\title{
TWO DIMENSIONAL FUNCTIONALLY GRADED \\ MATERIAL FINITE THICK HOLLOW CYLINDER AXISYMMETRIC VIBRATION MODE SHAPES ANALYSIS BASED ON EXACT ELASTICITY THEORY
}

\author{
MASOUd ASGaRI \\ Faculty of Mechanical Engineering, K. N. Toosi University of Technology, \\ Pardis Street, Molla-Sadra Avenue, Vanak Square, Tehran, Iran, \\ e-mail: asgari@kntu.ac.ir
}

[Received 09 December 2013. Accepted 09 March 2015]

\begin{abstract}
A thick hollow cylinder with finite length made of twodimensional functionally graded material (2D-FGM) is considered and its natural modes are determined, based on great importance of mode shapes information in order to understand vibration behaviour of structures. Three dimensional theory of elasticity implemented for problem formulation, since mode shapes of a thick cylinder are three dimensional even with axisymmetric conditions. The axisymmetric conditions are assumed for the 2D-FGM cylinder. The material properties of the cylinder are varied in the radial and axial directions, with power law functions. Effects of volume fraction distribution on the different types of symmetric mode shapes configuration and vibration behaviour of a simply supported cylinder are analyzed. Three dimensional equations of motion are used and the eigen value problem is developed, based on direct variation method.

KEY worDs: 2D-FGM, finite hollow cylinder, vibration mode shapes, elasticity theory.
\end{abstract}

\section{Introduction}

Recently, the composition of several different materials is often used in structural components, in order to optimize the responses of structures, subjected to severe loadings. The transition between different materials is performed gradually for reducing the local stress concentrations induced by abrupt transitions in material properties. This idea leads to the concept of functionally graded materials (FGMs) [1]. The mechanical properties of FGMs 
vary continuously between several different materials. Most of researches in this area are concerned with the thermo-elastic and residual stress analysis. In many applications of these materials, the vibration characteristics are of a great importance in addition to stress considerations. The vibration of FGM cylindrical structures has been studied by a number of researchers. Different studies on the vibration of cylindrical shells produced of a FGM, based on Love's and some other classical shell theories, have been performed and usually Rayleigh-Ritz and finite element methods, used for solving governing equations [2-5]. All of the previous discussed papers are focused on cylindrical shells, using the classical equations of thin shell theories, while the vibration behaviour of a thick walled cylinder is of considerable engineering importance. Very little studies have been revealed for thick hollow circular cylinders. They require a 3-D analysis, which is based on the theory of elasticity. As a result, in the literatures, the study of free vibrations of thick circular cylinders using three-dimensional theory of elasticity is relatively scarce in comparison to the study of thin shells, using other shell theories. Studies on shells, based on three-dimensional theory of elasticity, have been presented by some researchers for infinitely long cylindrical shells [6-9]. For finite-length thick cylindrical shells, different methods, such as finite element method, series solution, the Ritz energy method are used by some researchers for both solid and hollow homogeneous cases [10-13].

Most of the referred studies considered ways of determining the frequencies of cylinders. Few of the authors give a description of the mode shapes of the thick cylinders. While mode shapes are also very important sources of information for understanding and controlling the vibration of a structure [16]. Singal and Williams [14-15] combined experimental results with a Ritz energy method of analysis to compare frequencies for free-free cylinders. They gave a description for the mode shapes of thick-walled hollow cylinders and rings. Singhal et al. [16] presented theoretical and experimental modal analysis by using a thick-walled circular cylinder model to obtain its natural frequencies and mode shapes. On the other hand, in the previous discussed literature, vibration analysis of moderately thick-walled hollow cylinders are limited to isotropic and laminated cylinders and functionally graded thick hollow cylinders with finite length, were not seen in the literatures. Also, the functionally graded cylindrical shells considered are using thin shell theories. So, investigation of functionally graded thick hollow cylinder can be of a great importance. Also, in all of the discussed cases the variation of volume fraction and properties of the FGMs are one-dimensional and properties vary continuously from inner surface to the outer one with a prescribed function. But, conventional function- 
ally graded material may also not be so effective in some design problems since all outer or inner surfaces will have the same composition distribution, while in advanced machine elements, load distribution may change in two or three directions [17]. Therefore, more effective material resistance can be obtained, if the FGM has two-dimensional dependent material properties. Based on this fact, a two-dimensional FGM, whose material properties are bi-directionally dependent, is introduced. Recently, a few authors have investigated 2D-FGM especially its stress analysis [18-21].

The author also has investigated the natural frequencies of a thick hollow cylinder with finite length made of 2D-FGM [22]. The influence of constituent volume fractions on natural frequencies was studied by varying the volume fractions of the constituent metals and ceramics. Furthermore, the effects of length and thickness of the cylinder on the fundamental natural frequency were considered in different types of 2D-FGM cylinder. So far, investigation of mode shapes configuration of FGM thick finite cylinder has not be considered in previous studies. So, in order to extend the previous studies and investigating mode shape configuration of FGM thick hollow cylinder in this study, the free vibration analysis and all types of mode shapes of a thick hollow cylinder with finite length, made of 2D-FGM, on the basis of three-dimensional theory of elasticity is considered. The material properties of the cylinder are varied in the radial and axial directions with power law functions. Effects of volume fraction distribution and FGM configuration on the natural frequencies and three dimensional mode shapes of a simply supported functionally graded thick hollow cylinder are analyzed. The influence of constituent volume fractions is studied by varying the volume fractions of the constituent metals and ceramics. A functionally graded cylinder with two-dimensional gradation of distribution profile has been investigated, as well as the one-dimensional gradation of material distribution. Three dimensional equations of motion are used and the eigen value problem is developed based on Rayleigh-Ritz variation method and all axisymmetric and non-axisymmetric mode shapes are considered. Finite element method with graded material characteristics, within each element of the structure, is used for solution. Using conventional finite element formulations such that the property field is constant within an individual element for dynamic problems leads to significant discontinuities and inaccuracies [23]. These inaccuracies will be more significant in 2-D FGM cases. On the other hand, by using graded finite element in which the material property field is graded continuously through the elements, accuracy can be improved without refining the mesh size [24-25]. Based on these facts, the graded finite element developed by the author [26], is used for present problem modelling. 


\section{Problem formulation}

In this section, volume fraction distributions in the two radial and axial directions are introduced. The 3D governing equations of motion in cylindrical coordinates are obtained and graded finite element is used for modelling the non-homogeneity of the material.

\section{cylinder}

\subsection{Volume fraction and material distribution in 2D-FGM}

In the conventional one dimensional functionally graded cylinder, the cylinder's material is graded through the radial direction. Significant advances in fabrication and processing techniques have made it possible to produce FGMs, using processes that allow FGMs with complex properties and shapes, including two-and three-dimensional gradients, using computer-aided manufacturing techniques. 2D-FGMs are usually made by continuous gradation of three or four distinct material phases, that one or two of them are ceramics and the others are metal alloy phases. The volume fractions of the constituents vary in a predetermined composition profile. Now we consider the volume fractions of 2D-FGM at any arbitrary point in the axisymmetric cylinder of internal radius $r_{i}$, external radius $r_{o}$, and finite length $L$ shown in Fig. 1 . In the present cylinder, the inner surface is produced of two distinct ceramics and the outer surface from two metals. $c 1, c 2, m 1$ and $m 2$ denote first ceramic, second ceramic, first metal and second metal, respectively.

The function of volume fraction distribution of each material can be

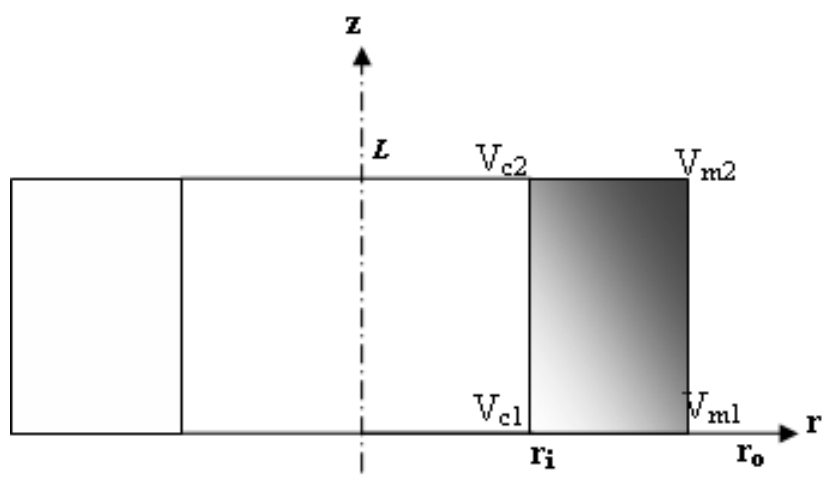

Fig. 1. Axisymmetric cylinder with two dimensional material distributions 
explained, as in [22]:

$(1 a-d)$

$$
\begin{aligned}
& V_{c 1}(r, z)=\left[1-\left(\frac{r-r_{i}}{r_{0}-r_{i}}\right)^{n_{r}}\right]\left[1-\left(\frac{z}{L}\right)^{n_{z}}\right], \\
& V_{c 2}(r, z)=\left[1-\left(\frac{r-r_{i}}{r_{0}-r_{i}}\right)^{n_{r}}\right]\left[\left(\frac{z}{L}\right)^{n_{z}}\right] \\
& V_{m 1}(r, z)=\left(\frac{r-r_{i}}{r_{0}-r_{i}}\right)^{n_{r}}\left[1-\left(\frac{z}{L}\right)^{n_{z}}\right] \\
& V_{m 2}(r, z)=\left(\frac{r-r_{i}}{r_{0}-r_{i}}\right)^{n_{r}}\left(\frac{z}{L}\right)^{n_{z}}
\end{aligned}
$$

where $n_{r}$ and $n_{z}$ are non-zero parameters, that represent the basic constituent distributions in $r$ and $z$ directions. Material properties at each point can be obtained by using the linear rule of mixtures, in which a material property, $P$, at any arbitrary point $(r, z)$ in the 2D-FGM cylinder is determined by linear combination of volume fractions and material properties of the basic materials, as in [22]:

$$
P=P_{c 1} V_{c 1}+P_{c 2} V_{c 2}+P_{m 1} V_{m 1}+P_{m 2} V_{m 2} .
$$

The basic constituents of the 2D-FGM cylinder are presented in Table 1.

Table 1. Basic constituents of the 2D-FGM cylinder

\begin{tabular}{|c|c|c|c|}
\hline Constituents & Material & $\mathrm{E}(\mathrm{Gpa})$ & $\rho\left(\mathrm{kg} / \mathrm{m}^{3}\right)$ \\
\hline$m 1$ & $\mathrm{Ti} 6 \mathrm{Al} 4 \mathrm{~V}$ & 2715 & 115 \\
\hline$m 2$ & $\mathrm{Al} 1100$ & 69 & 4515 \\
\hline$c 1$ & $\mathrm{SiC}$ & 440 & 3210 \\
\hline$c 2$ & $\mathrm{Al}_{2} \mathrm{O}_{3}$ & 150 & 3470 \\
\hline
\end{tabular}

It should be noted, that Poisson's ratio is assumed to be constant through the body. This assumption is reasonable, because of the small differences between the basic materials Poisson's ratios.

\subsection{Governing equations}

We consider a 2D-FGM thick hollow cylinder of internal radius, $r_{i}$ external radius $r_{o}$, and finite length $L$. Coordinates $r, z$ and $\theta$ are used in the 
analysis. Neglecting body forces, the Equations of motion in cylindrical coordinates are obtained, as:

$$
\begin{aligned}
& \frac{\partial \sigma_{r r}}{\partial r}+\frac{\partial \tau_{r z}}{\partial z}+\frac{\partial \tau_{r \theta}}{r \partial \theta}+\frac{\sigma_{r r}-\sigma_{\theta \theta}}{r}=\rho(r, z) \frac{\partial^{2} u}{\partial t^{2}}, \\
(2 a-c) \quad & \frac{\partial \tau_{r \theta}}{\partial r}+\frac{\partial \sigma_{\theta \theta}}{r \partial \theta}+\frac{\partial \tau_{z \theta}}{\partial z}+\frac{2 \tau_{r \theta}}{r}=\rho(r, z) \frac{\partial^{2} v}{\partial t^{2}}, \\
& \frac{\partial \tau_{r z}}{\partial r}+\frac{\partial \tau_{z \theta}}{r \partial \theta}+\frac{\partial \sigma_{z z}}{\partial z}+\frac{\tau_{r z}}{r}=\rho(r, z) \frac{\partial^{2} w}{\partial t^{2}},
\end{aligned}
$$

where $u, v$ and $w$ are radial, circumferential and axial components of displacement, respectively, those are functions of $(r, z, \theta, t)$ and $\rho(r, z)$ is the mass density, that depends on $r$ and $z$ coordinates. The constitutive equations for FGM are written as:

$$
\left\{\sigma_{i j}\right\}=[D(r, z)]\left\{\varepsilon_{i j}\right\},
$$

where the stress and strain components and the coefficients of elasticity are:

$$
\begin{gathered}
\left\{\sigma_{i j}\right\}=\left[\begin{array}{llllll}
\sigma_{r r} & \sigma_{\theta \theta} & \sigma_{z z} & \tau_{z \theta} & \tau_{r z} & \tau_{r \theta}
\end{array}\right] \\
\left\{\varepsilon_{i j}\right\}=\left[\begin{array}{llllll}
\varepsilon_{r r} & \varepsilon_{\theta \theta} & \varepsilon_{z z} & \gamma_{z \theta} & \gamma_{r z} & \gamma_{r \theta}
\end{array}\right] \\
{[D(r, z)]=\frac{E(r, z)}{(1+\nu)(1-2 \nu)}\left[\begin{array}{ccccccc}
1-\nu & \nu & \nu & 0 & 0 & 0 \\
\nu & 1-\nu & \nu & 0 & 0 & 0 \\
\nu & \nu & 1-\nu & 0 & 0 & 0 \\
0 & 0 & 0 & \frac{1-2 \nu}{2} & 0 & 0 \\
0 & 0 & 0 & 0 & \frac{1-2 \nu}{2} & 0 \\
0 & 0 & 0 & 0 & 0 & \frac{1-2 \nu}{2}
\end{array}\right],}
\end{gathered}
$$

where $\nu$ denotes the Poison's ratio, which is uniform through the cylinder and $E(r, z)$ is Young's modulus, that depends on $r$ and $z$ coordinates. The straindisplacement Equations are:

$$
\begin{gathered}
\varepsilon_{r}=\frac{\partial u}{\partial r}, \quad \varepsilon_{\theta}=\frac{\partial v}{r \partial \theta}+\frac{u}{r}, \quad \varepsilon_{z}=\frac{\partial w}{\partial z} \\
(8 a-c) \quad \gamma_{r z}=\frac{\partial u}{\partial z}+\frac{\partial w}{\partial r}, \quad \gamma_{z \theta}=\frac{\partial v}{\partial z}+\frac{\partial w}{r \partial \theta}, \quad \gamma_{r \theta}=\frac{\partial u}{r \partial \theta}+\frac{\partial v}{\partial r}-\frac{v}{r} .
\end{gathered}
$$


The cylinder is simply supported on its two end edges. So, mechanical boundary conditions on upper and lower edges are assumed as:

$$
v(r, 0, \theta, t)=v(r, L, \theta, t)=w(r, 0, \theta, t)=w(r, L, \theta, t)=0 .
$$

A solution that satisfies the circumferential displacement and defines a circular frequency is [27]:

$(10 a-c)$

$$
\begin{aligned}
& u(r, \theta, z, t)=\psi_{1}(r, z) \cos (m \theta) e^{i \omega t}, \\
& v(r, \theta, z, t)=\psi_{2}(r, z) \sin (m \theta) e^{i \omega t}, \\
& w(r, \theta, z, t)=\psi_{3}(r, z) \cos (m \theta) e^{i \omega t},
\end{aligned}
$$

where $m$ is the circumferential wave number and $\omega$ is the circular frequency. Also, considering the circumferential symmetry of the cylinder about the coordinate $\theta$, the displacement amplitude functions can be written as $\psi_{1}(r, z)$, $\psi_{2}(r, z)$ and $\psi_{3}(r, z)$. It is obvious that $m=0$ means the axisymmetric vibration. Certain specified uniform boundary conditions, along the two ends, can be satisfied by choosing the displacement amplitude functions properly.

\subsection{Graded finite element modelling}

The finite element method with graded element properties is used, in order to solve the governing equations. For this purpose, the variation formulation is considered. In conventional finite element formulations a predetermined set of material properties are used for each element, such that the property field is constant within an individual element. For modelling a continuously non-homogeneous material, the material property function must be discretized according to the size of elements mesh. This approximation can provide significant discontinuities. In addition, variation of material properties in two directions, such as the present problem, makes this effect more considerable. Based on these facts, the graded finite element is strongly preferable for modelling of the present problem.

The Hamilton's principle for the present problem is:

$$
\int_{t_{1}}^{t_{2}} \delta(\Pi-T) d t=0,
$$

where $\Pi$ and $T$ are potential and kinetic energy, respectively. These functions and their variations are: 


$$
\begin{gathered}
\delta T=\iiint_{v o l} \rho(r, z)\left(\left(\frac{\partial^{2} u}{\partial t^{2}}\right) \delta u+\left(\frac{\partial^{2} v}{\partial t^{2}}\right) \delta v+\left(\frac{\partial^{2} w}{\partial t^{2}}\right) \delta w\right) d V, \\
\delta \Pi=\frac{1}{2} \iiint_{v o l}\left\{\sigma_{i j}\right\}^{T}\left\{\delta \varepsilon_{i j}\right\} d V,
\end{gathered}
$$

where $V$ denotes the area and volume of the domain under consideration. Substituting Eqs. (12)-(13) in Hamilton's principle and applying side conditions, $\delta u\left(t_{1}\right)=\delta u\left(t_{2}\right)=\delta v\left(t_{1}\right)=\delta v\left(t_{2}\right)=\delta w\left(t_{1}\right)=\delta w\left(t_{2}\right)=0$, by part integration we have:

$$
\begin{aligned}
\iiint_{\text {vol }}\left\{\sigma_{i j}\right\}^{T} & \left\{\delta \varepsilon_{i j}\right\} d V \\
& +\iiint_{\text {vol }} \rho(r, z)\left(\left(\frac{\partial^{2} u}{\partial t^{2}}\right) \delta u+\left(\frac{\partial^{2} v}{\partial t^{2}}\right) \delta v+\left(\frac{\partial^{2} w}{\partial t^{2}}\right) \delta w\right) d V=0 .
\end{aligned}
$$

Four node tetrahedral element is are used to discrete the domain. By taking the nodal values of $u, v$ and $w$ as the degrees of freedom, a linear displacement model can be assumed, as:

$$
\left\{\begin{array}{c}
u \\
v \\
w
\end{array}\right\}^{e}=[N]\left\{Q_{0}^{e}\right\} e^{i \omega t}
$$

where $[N]$ is the matrix of assumed shape functions and satisfy certain specified boundary conditions and $\left\{Q_{0}^{e}\right\}$ is the nodal displacement vector of element. The cylinder will be divided into some brick-like subdivisions in radial, axial and circumferential directions and making tetrahedral mesh, through the use of brick subdivisions. In this case, $10 * 10 * 12$ brick subdivisions are produced, that lead to 2400 number of elements including 4356 degrees of freedom.

Using Eqs. (15) we can write:

$$
\{\varepsilon\}=[B]\left\{Q^{e}\right\},
$$


where components of matrix $[B]$ are as:

$$
[B]=\left[\begin{array}{cccc}
\frac{\partial N_{i}}{\partial r} \cos m \theta & 0 & 0 & \ldots \\
\frac{N_{i}}{r} \cos m \theta & \frac{m N_{i}}{r} \cos m \theta & 0 & \ldots \\
0 & 0 & \frac{\partial N_{i}}{\partial z} \cos m \theta & \ldots \\
0 & \frac{\partial N_{i}}{\partial r} \cos m \theta & -\frac{m N_{i}}{r} \sin m \theta & \ldots \\
\frac{\partial N_{i}}{\partial z} \cos m \theta & 0 & \frac{\partial N_{i}}{\partial r} \cos m \theta & \ldots \\
-\frac{m N_{i}}{r} \cos m \theta & \left(\frac{\partial N_{i}}{\partial r}-\frac{N_{i}}{r}\right) \sin m \theta & 0 & \ldots
\end{array}\right] .
$$

It follows, that matrix $[B]$ will have three additional columns for each node of the element. Applying Hamilton's principle for each element and substituting Equations (4) and (17), it can be achieved as:

$$
\begin{aligned}
& \delta\left\{Q^{e}\right\}^{T}\left[\iint_{V^{e}}[B(r, z, \theta)]^{T}[D(r, z)][B(r, z, \theta)] d V\right]\left\{Q^{e}\right\} \\
&+\delta\left\{Q^{e}\right\}^{T} {\left[\iiint_{V^{e}} \rho(r, z)[N]^{T}[N] d V\right]\left\{\ddot{Q}^{e}\right\}=0 }
\end{aligned}
$$

where $V^{e}$ is the volume of element.

In graded finite element, the interpolation function for the displacements within the elements and strain-displacement relations are the same as standard conventional finite element, as explained in Eqs. (17). In this way, the constitutive relation is:

$$
\left\{\sigma_{i j}\right\}=[D(r, z)]\left\{\varepsilon_{i j}\right\},
$$

where the components of $[D(r, z)]$ could be explicit functions, describing the actual material property gradient in which $E(r, z)$ is determined at each point through the element using distribution function of this property, based on rule of mixtures as:

$$
E(r, z)=E_{c 1} V_{c 1}(r, z)+E_{c 2} V_{c 2}(r, z)+E_{m 1} V_{m 1}(r, z)+E_{m 2} V_{m 2}(r, z) .
$$


Also, the mass density $\rho(r, z)$ is, in general, a function of position, as well as the mechanical properties. Therefore, in the graded finite element, the mass density distribution should be assigned into the element formulation as:

$$
\rho(r, z)=\rho_{c 1} V_{c 1}(r, z)+\rho_{c 2} V_{c 2}(r, z)+\rho_{m 1} V_{m 1}(r, z)+\rho_{m 2} V_{m 2}(r, z) .
$$

Since $\delta\left\{Q^{e}\right\}$ is the variation displacement of the nodal points and is arbitrary, it can be omitted from Eq. (18), and then this equation can be written as:

$$
[M]^{e}\left\{\ddot{Q}^{e}\right\}+[K]^{e}\left\{Q^{e}\right\}=0
$$

where the characteristic matrices are given as:

$$
\begin{aligned}
{[K]^{e} } & =\iiint_{V^{e}}[B(r, z, \theta)]^{T}[D(r, z)][B(r, z, \theta)] d V, \\
{[M]^{e} } & =\iiint_{V^{e}}[N(r, z, \theta)]^{T}[N(r, z, \theta)] \rho(r, z) d V .
\end{aligned}
$$

The integral must be taken over the elements' volume considering Eqs. (32) and (33), for finding the components of characteristic matrices. As $[D(r, z)]$ and $\rho(r, z)$ are not constant, these matrices are evaluated by numerical integration for each element. The integration calculated is using four Gaussian points.

Now, by assembling the element matrices, the global matrix equation for the structure can be obtained as:

$$
[M]\{\ddot{Q}\}+[K]\{Q\}=\{0\} .
$$

Once, the finite element equations are established, substituting $Q=$ $Q_{0} e^{i \omega t}$ into Eq. (24) will result in an eigen value equation, that can be solved using standard eigen value extraction procedures.

To get a better illustration of the mode shapes, the numerical results of the displacements relate to each eigen value on each node were transferred into the proper coordinate system and the radial, tangential, and longitudinal directions of the nodes were determined. 


\section{Numerical results and discussion}

To verify the present solution, as similar works to the present work are few, a finite length homogeneous thick cylinder, that can be found with the existing literature is used. A finite element model for axisymmetric elasticity is formulated directly in the cylindrical coordinates to study the vibration of hollow, isotropic and homogeneous finite length cylinders and frequencies are computed for free-free end boundary conditions in the reference [27] and compared with the reference [13]. For solving the mentioned problem by graded finite element method developed here, we consider a thick hollow cylinder with freely supported end conditions in which the material distribution is uniform. Therefore, the volume fraction exponent and property coefficients in the 2DFGM are taken as: $n_{z}=0, n_{r}=0, P_{c 1}=P_{c 2}=P_{m 1}=P_{m 2}=P$, where $P$ is single a uniform material properties of the cylinder. Comparison of the results for this case in Table 2 shows good agreement between two methods, again.

A thick hollow cylinder of inner radius $r_{i}=0.5 \mathrm{~m}$, outer radius $r_{o}=1$ $\mathrm{m}$ and length $L=2 \mathrm{~m}$ is considered. Effects of volume fraction distribution on the natural frequencies and mode shapes configuration of a simply supported functionally graded thick hollow cylinder are analyzed. The influence of constituent volume fractions are studied by varying the volume fractions of the constituent metals and ceramics. A functionally graded cylinder with twodimensional gradation of distribution profile has been investigated as well as the case, where the axial power law exponent is assumed to be zero, i.e., $n_{z}=0$, and the results of one-dimensional gradation of material distribution can be obtained in the hollow cylinder. The basic materials are as well explained in the

Table 2. Dimensionless frequencies $\Phi=(\omega H / \pi) \sqrt{\rho / G}$ of axisymmetric vibration for freely supported isotropic cylinders $(m=0, H / R=0.4, \nu=0.3)$

\begin{tabular}{|c|c|c|c|c|c|c|c|}
\hline$H / L$ & Method & $\Phi_{1}$ & $\Phi_{2}$ & $\Phi_{3}$ & $\Phi_{4}$ & $\Phi_{5}$ & $\Phi_{6}$ \\
\hline \multirow{2}{*}{0.2} & (Armenakas et al., 1969) & 0.20495 & 0.34765 & 1.07205 & 1.82336 & 2.09588 & 3.00073 \\
& (Cheung and Wu, 1972) & 0.20495 & 0.34765 & 1.07312 & 1.82688 & 2.10257 & 3.02850 \\
& Present method & 0.20492 & 0.34763 & 1.07309 & 1.82686 & 2.10254 & 3.02848 \\
\hline \multirow{2}{*}{0.4} & (Armenakas et al., 1969) & 0.27540 & 0.67185 & 1.23591 & 1.76178 & 2.25224 & 2.99980 \\
& (Cheung and Wu, 1972) & 0.27544 & 0.67188 & 1.23712 & 1.76587 & 2.25874 & 3.02742 \\
& Present method & 0.27539 & 0.67182 & 1.23704 & 1.76580 & 2.25868 & 3.02735 \\
\hline \multirow{2}{*}{0.6} & (Armenakas et al., 1969) & 0.42022 & 0.98133 & 1.44860 & 1.75005 & 2.43159 & 3.00691 \\
& (Cheung and Wu, 1972) & 0.42038 & 0.98145 & 1.45008 & 1.75433 & 2.43851 & 3.03437 \\
& Present method & 0.42032 & 0.98138 & 1.45001 & 1.75421 & 2.43842 & 3.03428 \\
\hline
\end{tabular}


previous section. Constituent materials are two distinct ceramics and two distinct metals described in Table 1 and Poison ratio $\nu=0.3$. Volume fractions of materials are distributed according to Eqs. $(1 a-d)$. Vibration characteristics of cylinder for some different powers of material composition profile $n_{r}$ and $n_{z}$ are presented and compared. Dimensionless frequency parameter is assumed as $\Omega=\left(r_{o} \omega\right) \sqrt{\rho / G}$.

The longitudinal and radial modes are uncoupled from pure torsion modes, when the circumferential wave number is taken as $m=0$ [17]. The symmetric modes in an isotropic cylinder are indicated in Table 3.

Table 3. Symmetric modes in isotropic cylinder, $n_{z}=0, n_{r}=0$

\begin{tabular}{|c|c|c|c|}
\hline $\begin{array}{c}\text { Longitudinal } \\
\text { mode }\end{array}$ & Mode 2 & Mode 3 & Mode 4 \\
\hline & 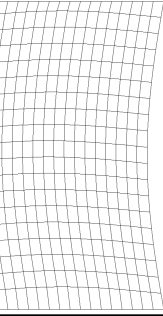 & \begin{tabular}{|l} 
MIIIAHIIA \\
\\
\\
\\
\\
\\
\\
\\
\end{tabular} & AIII \\
\hline
\end{tabular}

In order to investigate the effect of material distribution profile in the case of symmetrical modes, some selected mode shapes are computed for comparison, are shown in Tables 4 and 5. Three longitudinal-radial mode shapes corresponding to $m=0$ for different values of radial power exponent and zero axial power exponent are shown in Table 4. As the radial power exponent increases, the curvature in symmetric mode shapes decreases. It is more significant on the inner surface of the cylinder. Longitudinal-radial symmetric mode shapes, corresponding to $m=0$ for different non-zero values of radial and axial power exponents are shown in Table 5. It can be seen, that the influence of the constituent volume fractions on the symmetric mode shapes configuration is more significant on the inner surface and bottom edge of the cylinder. On the other radial power exponent has a more considerable effect.

Mode shapes are according to the classification of the modes of thick cylinders used by Wang and Williams [15].

Axial bending modes; in which the circumferential cross section segments bend oppositely in the axial direction, Radial motion with radial shearing modes; that for this kind the cylinder no longer retains a constant cross sectional along its length, Circumferential; in these modes adjacent segmental 
Table 4. Symmetric modes $(m=0)$ for various radial power law exponents in 1D-FGM cylinder

\begin{tabular}{|c|c|c|c|}
\hline $\begin{array}{l}\text { Longitudinal } \\
\text { mode }\end{array}$ & Mode 2 & Mode 3 & Mode 4 \\
\hline$n_{r}=0.2$ & 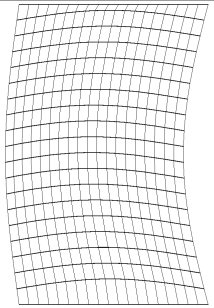 & $\begin{array}{l} \\
\\
\\
\\
\\
\\
\\
\\
\end{array}$ & 㖆 \\
\hline$n_{r}=0.5$ & 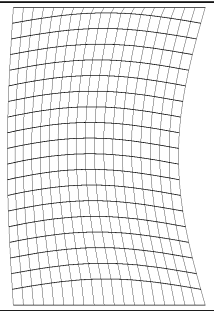 & $\begin{array}{l} \\
\\
\\
\\
\\
\\
\\
\\
\end{array}$ & 册地册我 \\
\hline$n_{r}=2$ & 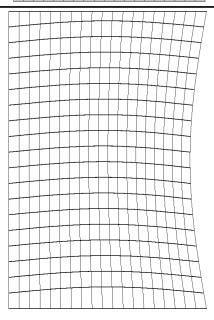 & 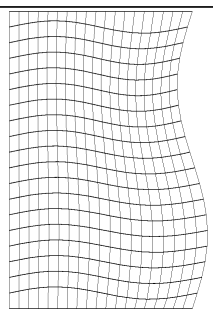 & 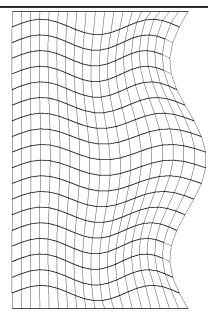 \\
\hline
\end{tabular}

elements expand or contract one by one in the circumferential direction. The median circumferential length of an expanding segment becomes longer and the length of the contracting segment becomes shorter and Global modes; for these modes the thick cylinder can be considered to behave as a simple beam vibrating in a transverse direction, a bar vibrating in torsion, or as a rod vibrating in a longitudinal direction. Although, displacements of each nodes of the cylinder are calculated, because more clear illustration just some cross sections of the cylinder on inner and outer radii are shown. The variations of the natural dimensionless frequencies parameter with the circumferential wave numbers, $m$ for different values of radial power exponent, while the axial power exponent $n_{z}=0$ is shown in Fig. 2. It is clear, that effect of variation of radial power exponent is more considerable for higher natural frequencies.

Effect of variation of axial power exponent for natural frequency is 
Table 5. Symmetric modes $(m=0)$ for various power law exponents in 2D-FGM cylinder

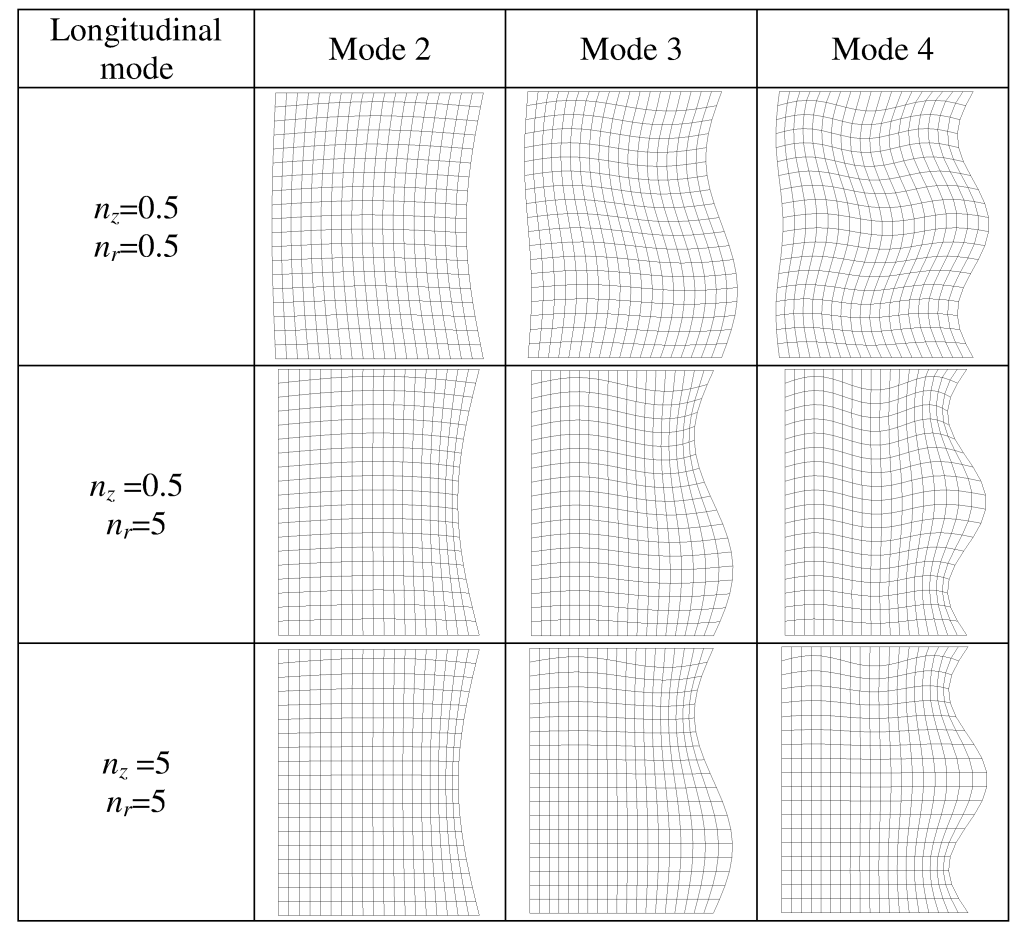

considered in Fig. 3. It is clear, that effect of axial power exponent on the natural frequencies is insignificant, when the radial power exponent is zero.

Variation of natural frequencies with circumferential wave number for different values of axial power exponent, while the radial power exponent is not zero $\left(n_{r}=2\right)$ is shown in Fig. 4 . The influence of the value of $n_{z}$ can be seen from this figure. It is clear, as $n_{z}$ increased, the natural frequencies increased, the natural frequencies and the mode shapes of different kinds of modes vary with changing material distribution profile.

\section{Conclusion}

A study on the free vibration of simply supported thick hollow cylinder with finite length made of 2D-FGM is presented, based on the three dimensional mode shapes. Material properties are graded in the thickness and longitudinal directions of the cylinder according to a volume fraction power law distribution. The equations of motion are based on three-dimensional elasticity theory and graded finite element method, which has some advantages to the 


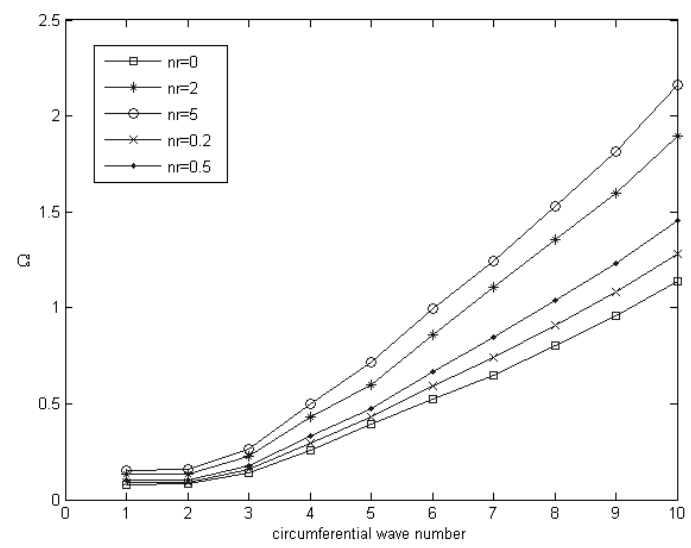

Fig. 2. Natural frequencies for various radial power law exponents in 1D-FGM cylinder, $n_{z}=0$

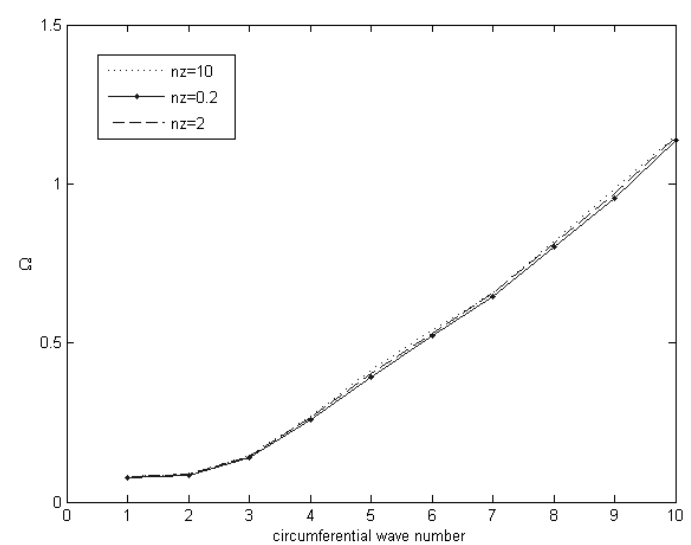

Fig. 3. Natural frequencies for various axial power law exponents in 1D-FGM cylinder, $n_{r}=0$

conventional finite element methods is employed for the solution. The effects of two-dimensional material distribution on the mode shapes characteristics are considered and compared with conventional one-dimensional FGM. Variation of the symmetric mode shapes against volume fraction power exponents in radial and axial directions is investigated. Variation of natural frequencies and symmetric mode shapes with circumferential wave number, associated with the numerous values of radial and axial power exponents are calculated 


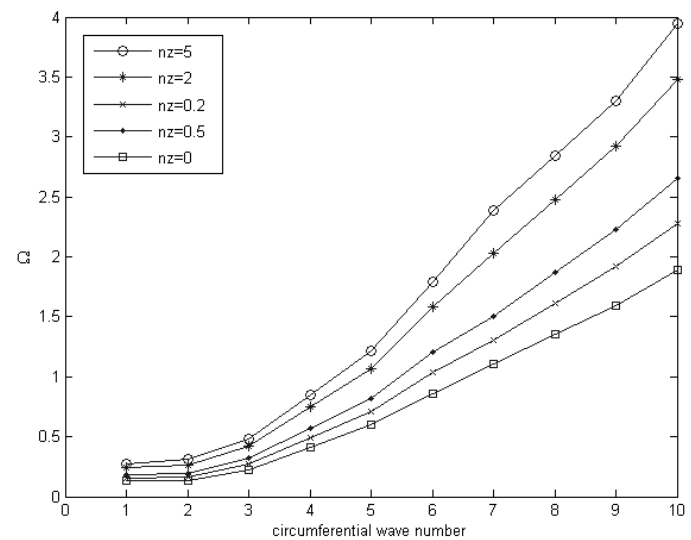

Fig. 4. Natural frequencies for various axial power law exponents in 2D-FGM cylinder, $n_{r}=2$

for 1D-FGM cylinder, as well as 2D-FGM cylinder. The study shows that the 2D-FGM cylinder exhibit interesting frequency and mode shape characteristics, when the constituent volume fractions are varied. Based on the achieved results, 2D-FGMs have a powerful potential for designing and optimization of structures, under multi-functional requirements.

\section{REFERENCES}

[1] Kolzumi, M. The Concept of FGM Ceramic Transaction. Functionally Graded Materials, 34 (1993), 3-10.

[2] Loy, C. T., K. Y. Lam, J. N. Reddy. Vibration of Functionally Graded Cylindrical Shells. Int Journal of Mechanical Sciences, 41 (1999), 309-324.

[3] Pradhan, S. C., C. T. Loy, K. Y. Lam, J. N. Reddy. Vibration Characteristics of Functionally Graded Cylindrical Shells under Various Boundary Conditions. Applied Acoustics, 61 (2000), 111-129.

[4] Zhao, X., T. Y. NG, K. M. Liew. Free Vibration of Two-side Simply-supported Laminated Cylindrical Panels via the Mesh-free Kp-Ritz Method. Int. Journal of Mechanical Sciences, 46 (2004), 123-142.

[5] Patel, B. P., S. S. Gupta, M. S. Loknath, C. P. Kadu. Free Vibration Analysis of Functionally Graded Elliptical Cylindrical Shells using Higher-Order Theory. Composite Structures, 69 (2005), 259-270. 
[6] Greenspon, J. E. Flexural Vibrations of a Thick-walled Cylinder According to the Exact Theory of Elasticity. Journal of Aerospace Sciences, 27 (1957), 13651373.

[7] Gazis, D. C. Three-dimensional Investigation of the Propagation of Waves in Hollow Circular Cylinders. Journal of the Acoustical Society of America, 31 (1959), 568-578.

[8] Nelson, R. B., S. B. Dong, R. D. KalRa. Vibrations and Waves in Laminated Orthotropic Circular Cylinders. Journal of Sound and Vibration, 18 (1971), 429444.

[9] Armenakas, A. E., D. S. Gazis, G. Herrmann. Free Vibrations of Circular Cylindrical Shells, Oxford, Pergamon Press, 1969.

[10] Gladwell, G., D. K. Vijay. Natural Frequencies of Free Finite-length Circular Cylinders. Journal of Sound and Vibration, 42 (1975), No. 3, 387-397.

[11] Gladwell, G., D. K. Vijay. Vibration Analysis of Axisymmetric Resonators. Journal of Sound and Vibration, 42 (1975), No. 2, 137-145.

[12] Hutchinson, J. R., S. A. El-Azhari. Vibrations of Free Hollow Circular Cylinders. Journal of Applied Mechanics 53 (1986), No. 2, 641-646.

[13] So, J., A. W. Leissa. Free Vibrations of Thick Hollow Circular Cylinders from Three-dimensional Analysis. Trans ASME, Journal of Vibrations and Acoustics, 119 (1997), 89-95.

[14] Singal, R. K., K. Williams. A Theoretical and Experimental Study of Vibrations of Thick Circular Cylindrical Shells and Rings. Trans ASME, Journal of Vibrations, Acoustics, Stress, and Reliability in Design, 110 (1988), 533-537.

[15] Wang, H., K. Williams. Vibrational Modes of Thick Cylinders of Finite Length. Journal of Sound and Vibration, 191 (1996), No. 5, 955-971.

[16] Singhal, R. K., W. Guan, K. Williams. Modal Analysis of a Thick-walled Circular Cylinder. Mechanical Systems and Signal Processing, 16 (2002), No. 1, $141-153$.

[17] Nemat-Alla, M. Reduction of Thermal Stresses by Developing Two Dimensional Functionally Graded Materials. Int. J. Solids Structures, 40 (2003), 73397356.

[18] Abudi, J., M. J. Pindera. Thermoelastic Theory for the Response of Materials Functionally Graded in two Directions. Int. J. Solids Structures, 33 (1996), 931966.

[19] Asgari, M., M. Akhlaghi, S. M. Hosseini. Dynamic Analysis of TwoDimensional Functionally Graded Thick Hollow Cylinder with Finite Length under Impact Loading. Acta Mechanica, 208 (2009), 163-180.

[20] Asgari, M., M. Akhlaghi. Thermo-Mechanical Analysis of 2D-FGM Thick Hollow Cylinder using Graded Finite Elements. Advances in Structural Engineering, 14 (2011), No. 6, 1059-1073.

[21] Asgari, M., M. Akhlaghi. Transient Thermal Stresses in Two-dimensional Functionally Graded Thick Hollow Cylinder with Finite Length. Arch Appl Mech, 80 (2010), 353-376. 
[22] Asgari, M., M. Akhlaghi. Natural Frequency Analysis of 2D-FGM Thick Hollow Cylinder Based on Three Dimensional Elasticity Equations. European Journal of Mechanics A/Solids, 30 (2011), 72-81.

[23] Santare, M. H., P. Thamburaj, A. Gazonas. The Use of Graded Finite Elements in the Study of Elastic Wave Propagation in Continuously Nonhomogeneous Materials. Int J Solids Structures, 40 (2003), 5621-5634.

[24] Santare, M. H., J. Lambros. Use of a Graded Finite Element to Model the Behaviour of Non-homogeneous Materials. J. Appl. Mech., 67 (2000), 819-822.

[25] Kim, J. H., G. H. Paulino. Isoparametric Graded Finite Elements for Nonhomogeneous Isotropic and Orthotropic Materials. J. Appl. Mech., 69 (2002), 502-514.

[26] Asgari, M., M. Akhlaghi. Transient Heat Conduction in Two-Dimensional Functionally Graded Hollow Cylinder with Finite Length. Heat and Mass Transfer, 45 (2009), 1383-1392.

[27] Buchanan, G. R., C. B. Y. YiI. Effect of Symmetrical Boundary Conditions on the Vibration of Thick Hollow Cylinders. Applied Acoustics, 63 (2002), 547-566. 